\title{
Facial Soft Tissue Thickness in Forensic Facial Reconstruction: Is it enough if Norms Set?
}

\author{
Gayathri Ramesh ${ }^{{ }^{*}}$, Ramesh Nagarajappa ${ }^{2}$, Gadiputi Sreedhar ${ }^{3}$ and Masineni Narayanappa Sumalatha ${ }^{4}$ \\ ${ }^{1}$ Department of Oral and Maxillofacial Pathology, Rama Dental College Hospital and Research Centre, Rama University, Kanpur, India \\ ${ }^{2}$ Department of Public Health Dentistry, Institute of Dental Sciences, Kalinga Nagar, Bhubaneswar, India \\ ${ }^{3}$ Department of Oral and Maxillofacial Pathology, BBD Dental College, BBD University, Lucknow, India \\ ${ }^{4}$ Department of Oral and Maxillofacial Diagnosis Radiology, BBD Dental College, BBD University, Lucknow, India
}

*Corresponding author: Dr. Gayathri Ramesh, Professor, Department of Oral and Maxillofacial Pathology, Rama Dental College Hospital and Research Centre, Kanpur, Uttar Pradesh, India, E-mail: amug3r@yahoo.co.in

Rec date: July 15, 2015 Acc date: August 17, 2015 Pub date: August 24, 2015

Copyright: $\odot 2015$ Ramesh $\mathrm{G}$ et al. This is an open-access article distributed under the terms of the Creative Commons Attribution License, which permits unrestricted use, distribution, and reproduction in any medium, provided the original author and source are credited.

\begin{abstract}
Facial reconstruction relies on the relationships between the facial features, subcutaneous soft tissues and underlying bony structure of the skull. If there is no clue for potential identity because of impossibility to compare questioned remains with possible familiar material, in such cases one of the last chances is to recreate ante mortem appearance by face reconstruction. Many of the soft tissue thickness values are significantly different from those reported for comparable groups, suggesting that individuals from different geographical areas have unique facial features thus requiring population-specific values. This literature review attempts to throw some light on the important aspects that has to be taken into consideration while setting norms of soft tissue thickness among various populations. To name a few are the differences among different skeletal malocclusions, differences in soft tissue thickness and the rate of tissue change among both men and women at different age groups.
\end{abstract}

Keywords: Forensic science; Facial; Malocclusion; Reconstruction; Skeletal; Soft tissue thickness

\section{Introduction}

Identification of human remains has been a major problem for the medico-legal system. Detailed examination of recovered unknown skeletal remains answers the questions about basic characteristics such as sex, age, and ethnicity and overcome traumas. Studying the unknown skeletal remains mostly becomes the only way to find out more information about individual characteristics, which could lead to identification of potential victim. If there is no clue for potential identity the most precise comparative techniques fail, because of impossibility to compare questioned remains with possible familiar material. In such cases one of the last options is to recreate ante mortem appearance by face reconstruction [1]. Following the first introduction of reconstruction in the late 19th century, other techniques have been developed including radiography, 3D statistical model, ultrasound, MRI, CT and Cone Beam Computed Tomography (CBCT) [2].

Facial contours were traditionally considered to be the result of positioning of underlying hard tissue followed by the soft tissue. However, current trend shows a paradigm shift from the conventional hard tissue analysis to also include both hard- and soft-tissue analysis [3]. For facial reconstruction standard soft tissue thickness (STT) values and other anatomy-based rules are used. STT values are commonly used to determine the amount or the depth of the tissues that falls on certain predetermined landmarks of the skull. This aids in the reconstruction of the face by giving a limit to work from when developing the initial face shape in the early stages of the reconstruction procedure. However, one of the problems associated with Forensic Facial Reconstruction (FFR) is the reliance on the use of these average facial STT's, as well as the difficulty of estimating these values [4].

This identification technique is based on the removal of skull bone fragments, subsequent fixation of soft tissue onto a universal commercial polystyrene head model, precise suture of dermatomuscular flaps, and definitive adjustment using cosmetic treatments. After reconstruction, identifying marks such as scars, eyebrows, facial lines, facial hair and partly hairstyle become evident. It is then possible to present a modified picture of the reconstructed face to relatives. After comparing the results with photos of the person before death, this technique has proven to be very useful for identifying unknown bodies when other identification techniques are not available [5]. This reconstruction method being rather quick and especially for its results can be recommended as an auxiliary technique in the identification of unknown victims with crushing head injuries and in the evaluation of devastating gunshot wound of the head [6].

Hence this literature review attempts to compile the shortcomings in assessing STT and sheds some light on the important aspects to be considered while setting norms of STT among various populations. A total of 708 potentially relevant records were found in the databases (PubMed, Cochrane Library and Google Scholar), dated between 1970 to July 2015.

\section{Discussion}

For the purpose of individual identification Forensic Facial Reconstruction (FFR) is an attempt to reproduce a likeness of the facial features of an individual, based on characteristics of the skull [4]. Until these days no agreement exists upon the number of chosen landmarks, name of the landmarks and also their correct position. In 
several cases the variable authors call the same landmarks in different ways [7].

Description of the anatomical landmarks used for soft tissue (ST) depth measurement according to De Greef et al., were as follows [8]:

\section{Midline landmarks}

- Supraglabella - Most anterior point on midline

- Glabella - Cross-point between midline and supraorbital line

- Nasion - Midpoint of the fronto-nasal suture

- End of nasal - Junction between bone and cartilage of the nose

- Mid-philtrum - Centered between nose and mouth on midline

- Upper lip - Midline on the upper lip

- Lower lip - Midline on the lower lip

- Chin-lip fold - Midline centered in fold chin, below lips

- Mental eminence - Centered on most anteriorly projecting point of chin

- Beneath chin - The vertical measure of the ST on the most inferior point of the chin

\section{Bilateral landmarks}

- Frontal eminence - Centered on pupil, most anterior point of the forehead

- Supraorbital - Centered on pupil, just above eyebrow

- Lateral glabella - Junction of the frontal, maxillary, and lacrimal bones on the medial bone of the orbit Lateral nasal - Side of the bridge of the nose on the Frankfurt horizontal plane

- Suborbital - Centered on pupil, just under infra-orbital margin

- Inferior malar - Centered on pupil, just interior to zygomatic process

- Lateral nostril - Next to the most lateral point of the alar border

- Naso-labial ridge - The prominence either side of the philtrum

- Supracanina - Vertically lined up with the cheilion, on the horizontal level of the mid-philtrum

- Subcanina - Vertically lined up with the cheilion, on the horizontal level of the chin-lip fold

- Mental tubercle anterior - Most prominent point on the lateral bulge of the chin mound

- Mid-lateral orbit - Vertically centered on the orbit, next to the lateral orbital border

- Supraglenoid - Root of the zygomatic arch just anterior to the ear

- Zygomatic arch - Most lateral curvature of the zygomatic bone

- Lateral orbit - Lined up with the lateral border of the eye on the center of the zygomatic process

- Supra-M2 - Cheek region, lateral: lined up with nasal base; vertical: lined up beneath lateral border of the eye

- Mid-masseter - Middle of the masseter, the halfway point between the supraglenoid and the gonion Occlusal line - Border of the masseter, on vertical level of the cheilion

- Sub-M2 - Below the second molar on horizontally lined up with supra-M2

- Gonion - At the angle of the mandible

- Mid-mandibular angle - Inferior border of the mandible, vertically lined up with supra-M2.

Nowadays the number of surveys about facial soft tissue thickness is increasing. They differ from each other by selection of imaging technique and level of statistic evaluation. One of the most accurate measurements can be obtained by using CT (computed tomography) and MRI (Magnetic Resonance Imaging), but it belongs to invasive techniques because of radiation. Differences can be found also in number of landmarks, their position, as well as sample size [1].

Cephalometric norms for different ethnic and racial groups have been established previously. Studies are limited to establish whether the variation between sexes and ages within and between population groups may or may not be great enough to influence an identification or lack thereof. Most investigators have concluded that there are significant differences among these groups, and indicate that normal measurements for one group should not be considered normal for every other race or ethnic group. Therefore, it is important to develop individual standards for each population. Different racial groups must be treated according to their own characteristics [4].

Facial reconstruction in the forensic field uses one measure of mean thickness for each anthropometric point across all skeletal classes. Important point to be noted is that most of the investigators have mostly set STT norms for skeletal class 1 malocclusion pertaining to orthodontic treatment and not based for facial reconstruction.

When literature search was done pertaining to STT's database set for different skeletal malocclusion classes only few studies have investigated the soft tissue thickness of patients with different skeletal malocclusions. The published data was for women only- Japanese girls (aged 6-16 years) and women (aged 17-33 years) who had different skeletal malocclusions and one study for skeletal class III in South African females for forensic purpose $[4,9,10]$.

Utsuno et al. [10] found significant differences among skeletal classes at points such as, Subnasale, Labrale superious, Labiomentale, and Pogonion. Kamak and Celikoglub (2012)11 have determined STT differences among skeletal malocclusions for both men and women at the Labrale superious, Stomion, and Labrale inferius sites. The disagreement between the findings of these authors might be due to the racial differences. The greatest difference in soft tissue thickness was found between classes II and III, with class I being intermediate in Japanese females [10]. They also emphasized to use a measure of thickness for each skeletal class at points for which thickness differs significantly in order to obtain more accurate investigation results [10].

When difference in the STT between the genders is considered, statistically significant differences has been noted for the thickness at few landmarks such as, Labrale superius, Labrale inferius, Pogonion, and Menton [11]. The male group exhibited straighter profile, thicker lips, prominent nose, deep mentolabial sulcus, and a prominent chin than females. The differences could be considered in diagnosis and treatment planning for orthodontic practice and for orthognathic surgery [3]. Petra Panenková in her study has observed significant sex differences between the facial depths of men and women. In the majority of landmarks, males had thicker soft tissues than females. The only exception was landmark "lateral orbit", on zygomaticum, which showed the significant larger values in women. This fact indicate that while male and female means at craniofacial landmarks differ slightly, and even at statistically significant levels, individual male and female soft tissue depths are often the same or very similar [1].

Kamaka and Celikoglub [12] have noted statistically significant gender differences for all of the points in each skeletal class. In their observation it has been determined that, different mean ages show that the STT parameters are more statistically significant in men than in 
women. As humans age, the facial feature undergoes many changes that we recognize as aging. The significant changes occur around the midface region by the fourth decade [13]. Some of the distinctive features of an aged face include sunken volume around the periorbital region, the increasing prominence of the nasolabial fold and the accumulated folds of skin along the jaw. As the change in volume of subcutaneous fats at various facial points is found to be inconsistent, it suggests that there might be a combination of atrophy and migration of fats [14]. Plastic surgeon Val Lambros in his research for better clinical treatment of the aging face, presented that many facial landmarks are stable and does not descent with age [15].

The correlation between facial soft tissue thickness and age was also observed in the study by Petra Panenková mostly at forehead region, nose region, eyes and cheek region. The negative correlation with increasing age was observed at mouth zone. According to the author these differences were probably more influenced by body constitution than age [1]. The mandibular area showed greater differences between the right and left sides [16]. Perlaza Ruiz has reported no significant difference associated to gender for most anatomical landmarks in Colombian population. However, where differences were found, these were mainly located on the anatomical landmarks of the medial line and particularly with greater value among males [17].

According to Tng Ching Hwa [18] landmark located at the periorbital region, which supposedly has a sunken look on the aging face that one would normally associate with the atrophy of soft tissue. However the result shows that the soft tissue thickness decrease from the young to middle age group, and then increase from the middle to old age group. The change in the dimensions of the orbital rim might be one of the factors that offset the effect of atrophy and migration of soft tissue.

Wilkinson [19] stated that the age-related changes in tissues at the mouth and lower cheek tend to decrease with age, and tissues at the chin and eyebrow may increase with age. Similar results were obtained in a study by Greef et al. [20]. But with increase was observed in inferior malar and the mid mandibular angle and decrease in the occlusal line and mid masseter muscle. An explanation for these local differences attributed on the role of fat on the ageing face and they concluded that the ageing face can be analyzed as a change in volume and position of the different fat compartments, both superficial and deep [18].

Although the age had influence on facial soft tissue thickness, it is likely that weight would have bigger influence [1]. According to Tng Ching Hwa [18] one of the limitations of his study is that weight of the subjects is not included in the scope, hence the readings from a few subjects were observed to have either much larger or smaller thickness reading than the rest of their age group. Although few soft tissue depth variations with body weight and body mass index (BMI) have been reported, the use of different tissue depths with regard to body composition has minimal effect on the overall pattern of facial form. It significantly affects the subjective assessment, which suggests that variation in weight may be an important contributor to the ability to achieve correct recognition of a reconstructed face [10,21].

According to Greef et al. [20] the impact of the body composition, on facial STT depends on two important aspects: (a) all tissues are thinner for emaciated compared to well-nourished people with the exception of the nasal bridge, and (b) the landmarks differing most are the chin, mandible and cheek region. In his study the cheek and mandible zone were the most influenced by the BMI.
Stephan and Simpson [22] stated that the population differences that do exist (even the differences within population groups) are likely overpowered by the differences caused between different measurement methods, meaning that linked to the limited tissue thickness data is the lack of a standard method for determining these tissue thickness values and approximating facial features. Using different measurement methods, each with its own advantages, disadvantages and measuring errors, contributes to the variation that occur in STT data, since it affects the magnitude and accuracy of the obtained values, as well as the confidence with which the values can be regarded as accurate [4].

Aulsebrook and Van Rensburg [23] revealed that when strict adherence to the traditional tissue depths of White Europeans and Black Africans were utilized in the reconstruction of a skull of mixed racial origin, the accuracy of the facial approximation was compromised. Similarly Wilkinson [19] suggested that strict adherence to the exact tissue depths should be avoided and that the morphology of the skull and the anatomy should be followed with tissue depth measurements being used only as guides.

\section{Conclusion}

Hence to conclude there is also no way to tell which method provides the best resemblance of the true STT of humans, but refinement of the methods, additional data and larger samples, including regional variations, as well as practical and identification tests on this data, should further support the reliability of the soft tissue values used in reconstructions. It should also be remembered that prior to application of STT in facial reconstruction STT norms set among any population should consider different age groups including ageing changes, gender, different skeletal malocclusion classes, weight and body mass index, population selected should not be from mixed family at least for three generations and standard method for analysis. It will probably never be possible to describe and predict all the huge amount of face variations. Nevertheless, it is necessary to study relationships between cranial and facial features to obtain nearly acceptable resemblance to the victim.

\section{References}

1. Panenková $\mathrm{P}$, Beňuš R, Masnicová S, Obertová Z, Grunt J (2012) Facial soft tissue thicknesses of the mid-face for Slovak population. Forensic Sci Int 220: 293.

2. Masoume J, Farzad E, Mohaddeseh MM (2014) Facial Soft Tissue Thickness in North-West of Iran. Adv Biosci Clin Med. 3: 29-34.

3. Thomas M, Reddy VD, Lakshmi HV (2012) Soft-tissue cephalometric norms for the Lambada population in Telangana Region of Andhra Pradesh. Indian J Dent Res 23: 353-358.

4. Cavanagh D, Steyn M (2011) Facial reconstruction: soft tissue thickness values for South African black females. Forensic Sci Int 206: 215.

5. Joukal M, Frišhons J (2015) A facial reconstruction and identification technique for seriously devastating head wounds. Forensic Sci Int 252: 82-86.

6. Hejna P, Safr M, Zátopková L (2011) Reconstruction of devastating head injuries: a useful method in forensic pathology. Int J Legal Med 125: 587-590.

7. De Greef S, Claes P, Vandermeulen D, Mollemans W, Suetens P, et al. (2006) Large-scale in-vivo Caucasian facial soft tissue thickness database for craniofacial reconstruction. Forensic Sci Int 159 Suppl 1: S126-146.

8. Brown RE, Kelliher TP, Tu PH, Turner WD, Taister MA (2004) A survey of tissue-depth landmarks for facial approximation. Foren Sci Comm 6.

9. Utsuno H, Kageyama T, Uchida K, Yoshino M, Miyazawa H, et al. (2010) Facial soft tissue thickness in Japanese children. Forensic Sci Int 199: 109. 
Citation: Ramesh G, Nagarajappa R, Sreedhar G, Sumalatha MN (2015) Facial Soft Tissue Thickness in Forensic Facial Reconstruction: Is it enough if Norms Set?. J Forensic Res 6: 299. doi:10.4172/2157-7145.1000299

Page 4 of 4

10. Utsuno H, Kageyama T, Uchida K, Yoshino M, Oohigashi S, et al. (2010) Pilot study of facial soft tissue thickness differences among three skeletal classes in Japanese females. Forensic Sci Int 195: 165.

11. Utsuno H, Kageyama T, Uchida K, Kibayashi K (2014) Facial soft tissue thickness differences among three skeletal classes in Japanese population. Forensic Sci Int. 236: 175-180

12. Kamak H, Celikoglu M (2012) Facial soft tissue thickness among skeletal malocclusions: is there a difference? Korean J Orthod 42: 23-31.

13. Allan E Wulc, Pooja Sharma, Craig N Czyz (2012) Midfacial rejuvenation. Chapter 2, The anatomic basis of midfacial aging, Springer sciences and Business media 15-28.

14. Owsley JQ, Roberts CL (2008) Some anatomical observations on midface aging and long-term results of surgical treatment. Plast Reconstr Surg 121: $258-268$.

15. Lambros V (2007) Observations on periorbital and midface aging. Plast Reconstr Surg 120: 1367-1376.

16. Hwang HS, Park MK, Lee WJ, Cho JH, Kim BK, et al. (2012) Facial soft tissue thickness database for craniofacial reconstruction in Korean adults. J Forensic Sci 57: 1442-1447.
17. Perlaza Ruiz NA (2013) Facial soft tissue thickness of Colombian adults. Forensic Sci Int 229: 160

18. Tng Ching Hwa (2012-2013) Examination of facial soft tissue due to aging. National university of Singapore

19. Wilkinson C (2004) Forensic facial reconstruction. Chapter 2, Facial tissue depth measurement, Cambridge University press 124-156.

20. De Greef S, Vandermeulen D, Claes P, Suetens P, Willems G (2009) The influence of sex, age and body mass index on facial soft tissue depths. Forensic Sci Med Pathol 5: 60-65.

21. Dong Y, Huang L, Feng Z, Bai S, Wu G, et al. (2012) Influence of sex and body mass index on facial soft tissue thickness measurements of the northern Chinese adult population. Forensic Sci Int 222: 396.

22. Stephan CN, Simpson EK (2008) Facial soft tissue depths in craniofacial identification (part I): an analytical review of the published adult data. J Forensic Sci 53: 1257-1271.

23. Aulsebrook WA, Van Rensburg JHJ (1982) The significance of race determination in facial reconstruction. Abstract of lecture at the 20th Scientific Congress of International Association of Dental Research. J Dent Res 67: 783. 